\title{
Precise Radial Velocity Measurements with a Cassegrain Spectrograph, I: Wavelength calibration
}

\author{
I. V. Ilyin, R. Duemmler
}

Astronomy Division, University of Oulu, FIN-90571, Oulu, Finland

\begin{abstract}
We briefly describe the instrumental effects which affect the accuracy of the radial velocity measurements. We have implemented several methods to correct for the instability effects and improve the accuracy of the measurements. These include modifications of the observational strategy and a time-dependent wavelength solution as well as a discussion of the error of the offset from cross-correlation. These methods are applied to observations obtained with the high resolution échelle spectrograph SOFIN mounted at the Cassegrain focus of the alt-azimuth $2.56-\mathrm{m}$ Nordic Optical Telescope, La Palma, Canary Islands.
\end{abstract}

\section{Introduction}

To obtain the maximal achievable accuracy of the radial velocity measurements with a Cassegrain spectrograph we take into account the instrumental effects reviewed by Griffin \& Griffin (1973), and analyzed quantitatively by Brown (1990).

Bending of the spectrograph as the telescope and its adapter change the spatial orientation, liquid nitrogen evaporation which causes change of the weight of the CCD dewar attached, temporal variations of the ambient temperature, and the refractive index of the air result in a systematical drift of the spectral lines during one exposure. Assuming that during a short period of time these effects are small, progressive, and changing linearly, one would prefer to take external calibration lamp spectra before and after each stellar exposure, and to divide one long stellar exposure into a series of shorter ones, long enough to accumulate sufficient signal-to-noise, which makes the sum of systematic and random errors minimal. To reconstruct the expected position of the stellar lines in wavelengths at the mid-point of the exposure we use a wavelength solution based on the three-dimensional model described below.

A second-order effect of the drift is the broadening of spectral lines which would lead to spurious offsets of the radial velocity due to increased blending and distortions of the line profile. Fortunately, the effect is not too large if one considers that the progressive drift results in the convolution of spectral line profile with a rectangular profile. In our case, a drift by 0.3 pixels of a line of 2 pixels FWHM would increase its width by $1 \%$. The smallness of this effect implies that the effect on the radial velocities apart from the overall $\mathrm{drift}$ is negligible (see also Sect. 3.). 
The way of setting the star on the slit of the spectrograph coupled with the effective seeing (including the accuracy of the guiding) results in random deviations of the stellar line positions in wavelength from what was expected from the above correction. The effect comes from the non-uniform illumination of the slit which leads to an unequal filling of the collimator pupil and échelle, and is changing from one exposure to another. The effect becomes magnified by better seeing; less accurate guiding smears the stellar image on the slit (in our case, the projected slit width is $1860 \mathrm{~m} \mathrm{~s}^{-1}, 0.25$ on the sky, and the median seeing is 0.8 ). To eliminate this effect we use telluric lines as an external reference source.

Furthermore, the effects outlined above are wavelength dependent, which makes these wavelength corrections functions of the spectral order number. The wavelength solution eliminates this dependency for the comparison spectrum with sufficient accuracy (see Fig. 1). But the stellar radial velocities reveal a remaining trend across the orders resulting from the difference in the slit illumination. Telluric lines, if well distributed across the orders, can also be used to correct for this tilt; otherwise, the stellar spectra themselves are used.

\section{Wavelength solution}

In a series of comparison images, for each spectral order the positions of the Th-Ar comparison lines are determined by non-linear weighted least-squares fits of Gaussian profiles. Let $x_{i j}$ be the position along the CCD columns (the dispersion direction) of the line number $i$ of the image number $j, y_{i j}$ the position along the CCD rows, $k_{i j}$ the spectral order number, $\lambda_{i j}$ the wavelength, $t_{j}$ the time (mid-point of the exposure) since the first image. Let the cross-dispersion axis $k \lambda=$ const be tilted with respect to $C C D$ rows due to imperfection of the CCD alignment, diverging due to optical distortions, and changing linearly with time, so that the angle of the axis is:

$$
a_{i j}=b_{0}+b_{1} t_{j}+\left(d_{0}+d_{1} t_{j}\right) x_{i j} .
$$

Let $\Delta$ be the linear shift between images along $x$ per time unit; then a coordinate transformation gives the positions $u_{i j}$ in the undistorted system:

$$
u_{i j}=\left(x_{i j}+\Delta \cdot t_{j}\right) \cos a_{i j}+y_{i j} \sin a_{i j} .
$$

Let $k_{c}$ be the common order to which the wavelengths of all the others are translated, and $n$ the degree of the dispersion polynomial:

$$
\lambda_{i j} \frac{k_{i j}}{k_{c}}=\sum_{m=0}^{n} c_{m} u_{i j}^{m} .
$$

Therefore, the model to be fitted is

$$
\lambda=F\left(x, y, t, k, k_{c} ; \Delta, b_{0}, b_{1}, d_{0}, d_{1}, c_{0}, c_{1} \ldots, c_{n}\right) .
$$

All other possible parameters can be neglected for our spectrograph. This model is optimized by the Marquardt method for non-linear least-squares. To get a more convenient function to be used for rebinning, the above function is 

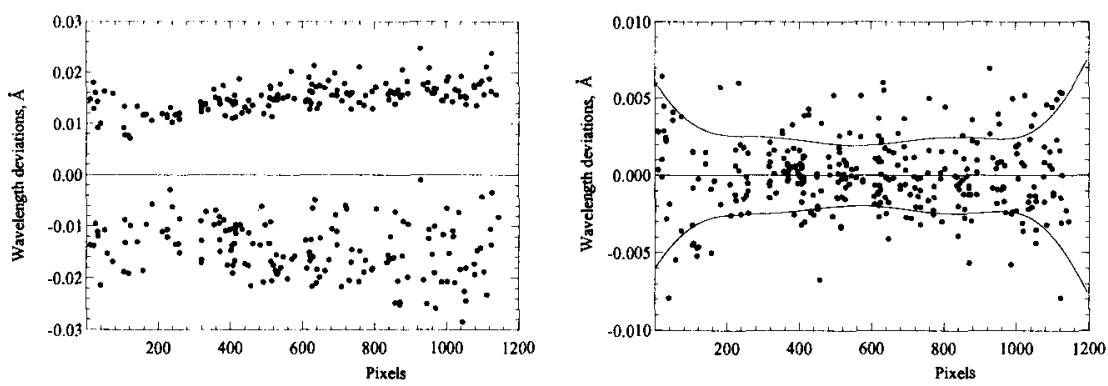

Figure 1. Wavelength solution of two comparison images selected to exaggerate the effects. Left: scatter plot of 363 spectral lines around a dispersion polynomial of 3rd degree prior to corrections. Right: after the optimization (the vertical scale enlarged three times) it converged to weighted $\mathrm{rms}=2.5 \mathrm{~m} \AA=130 \mathrm{~m} \mathrm{~s}^{-1}$ at $5635 \AA$. The two curves show the $10 \sigma$ level of the error of the fit as a function of the pixel number. The wavelength error in the image center is $\sigma=0.2 \mathrm{~m} \AA=10 \mathrm{~m} \mathrm{~s}^{-1}$ and steeply rises at the edges.

subsequently approximated by two-dimensional Chebyshev polynomials on the regular grid of $x$ as function of time for each order separately.

\section{The error of the cross-correlation offset determination}

For shift determination between spectra the cross-correlation method is used. A good knowledge of the error is essential, e.g. for proper weighting of the radial velocity measurements in orbital fits.

The offset $\delta$ between the program $p(x)$ and the template $t(x)$ spectrum is the offset at which the match between the spectra is best, i.e. the merit function

$$
\chi^{2}(a, b, \delta)=\sum_{i=0}^{N}\left(\frac{a+b t\left(x_{i}+\delta\right)-p\left(x_{i}\right)}{\sigma_{i}}\right)^{2}
$$

is minimized. Here, the free parameters $a$ and $b$ constitute the intensity transformation of the template to the program spectrum for each trial offset $\delta, \sigma_{i}$ is the uncertainty of the intensity of $p\left(x_{i}\right)$, and the index $i$ is ranging within the. overlapping region of the two spectra for the given $\delta$ with possible exclusion of intervals containing unwanted features in the programme spectrum; this implies that $N$, the number of overlapping pixels, is a function of the offset. The offset can be any fractional part of the step size which implies that the template spectrum has to be noiseless to allow interpolation between pixels.

The merit function can be expressed as a function of only one variable:

$$
\chi^{2}(\delta)=\left[1-r^{2}(\delta)\right] \cdot \sum \omega_{i}\left(p_{i}-\bar{p}\right)^{2}
$$


where the weighted cross-correlation function $r(\delta)$ is

$$
r(\delta)=\frac{\sum \omega_{i}\left(p_{i}-\bar{p}\right)\left(t_{i}(\delta)-\bar{t}(\delta)\right)}{\sqrt{\sum \omega_{i}\left(p_{i}-\bar{p}\right)^{2} \cdot \sum \omega_{i}\left(t_{i}(\delta)-\bar{t}(\delta)\right)^{2}}}
$$

with the notations: $\omega_{i}=1 / \sigma_{i}^{2}, t_{i}(\delta) \equiv t\left(x_{i}+\delta\right)$, where the weighted averages:

$$
\bar{p}=\frac{\sum \omega_{i} p_{i}}{\sum \omega_{i}} \text { and } \bar{t}(\delta)=\frac{\sum \omega_{i} t_{i}(\delta)}{\sum \omega_{i}} .
$$

The variances of the free parameters in least-squares is the inverse of the curvature matrix times the variance of the fit (Martin 1971):

$$
\sigma_{\delta}^{2}=2\left(\frac{d^{2} \chi^{2}(\delta)}{d \delta^{2}}\right)^{-1} \frac{\chi^{2}(\delta)}{N-2}
$$

which results in the formula:

$$
\sigma_{\delta}^{2}=-\left(\frac{d^{2} r}{d \delta^{2}}\right)^{-1} \frac{1-r^{2}}{r(N-2)}
$$

where the variance incorporates both the random noise of the spectrum and the systematic error due to template mismatch.

The position of the cross-correlation maximum and its curvature is found by fitting a polynomial of degree 3-4 to the very top of the peak of $r(\delta)$ which is calculated within an interval of a few pixels, re-sampled with a subpixel step size. Our Monte-Carlo simulations show a complete agreement with the above error estimation.

The uncertainty (10) of the position difference of two similar Gaussians, one being noiseless, the other being with a signal-to-noise ratio SNR, with the depths $d$ in continuum units, the widths FWHM, the sampling interval $\Delta \lambda$, becomes the well-known formula:

$$
\sigma_{\delta}=(2 \pi \ln 2)^{-1 / 4} \cdot \frac{\sqrt{\Delta \lambda \cdot \mathrm{FWHM}}}{r \cdot \mathrm{SNR} \cdot d} .
$$

In the case when a noiseless template spectrum is not available, $r(\delta)$ could still be calculated if $\delta$ varies with the step size of the program spectrum. Otherwise, a non-linear optimization of the full merit function, which includes the scaled uncertainties of the template spectrum, would be necessary to find the offset; the error of the offset then follows from the covariance matrix.

\section{Discussion}

Dravins: If you were to construct another high-resolution spectrograph for the Nordic Optical Telescope, would that again be a cassegrain one, or would you prefer some other focus position?

Ilyin: In my opinion it would be a Naysmyth focus spectrograph with an appreciable thermostabilization of its interior. 


\section{References}

Brown, T.M. 1990, ASP Conf. Ser. 8, 335

Griffin, R. \& Griffin, R. 1973, MNRAS 162, 243

Martin, B.R. 1971, Statistics for Physicists (New York: Academic Press) 